\title{
Modeling of Simultaneous Chemical and Phase Equilibria in Systems Involving Non-reactive and Reactive Azeotropes
}

Vassilis Koulocheris ${ }^{\mathrm{a}}$, Marina Pantelia ${ }^{\mathrm{a}}$ Eirini Petropoulou ${ }^{\mathrm{a}}$, Vasiliki Loulia $^{\mathrm{a}}$ Epaminondas Voutsas ${ }^{\mathrm{a}, *}$

${ }^{a}$ Laboratory of Thermodynamics and Transport Phenomena,

School of Chemical Engineering, National Technical University of Athens, 9, Heroon Polytechniou Str., Zografou Campus, 15780 Athens, Greece

\section{Supporting Information}

\footnotetext{
${ }^{*}$ Corresponding author.

E-mail address: evoutsas@chemeng.ntua.gr (E. Voutsas)
} 
Table S1. Mathias-Copeman parameters ${ }^{1}$

\begin{tabular}{cccc}
\hline Component & $\mathbf{C}_{\mathbf{1}}$ & $\mathbf{C}_{\mathbf{2}}$ & $\mathbf{C}_{\mathbf{3}}$ \\
\hline Methanol & 1.20979 & -0.11342 & -0.88667 \\
MTBE & 0.73832 & 0.17566 & -0.05901 \\
Acetic acid & 1.22453 & -1.5764 & 2.41004 \\
Isopropanol & 1.18454 & 0.69615 & 0.03812 \\
Water & 0.92366 & -0.37937 & 0.44243 \\
Isopropyl acetate $^{\mathrm{a}}$ & 0.84758 & 0.52717 & -0.94506 \\
\hline
\end{tabular}

${ }^{a}$ Determined in this work

Table S2. Experimental phase equilibrium data employed in this work

\begin{tabular}{cccccc}
\hline System & Ref. & Type $^{\mathbf{a}}$ & ND $^{\mathbf{b}}$ & T range (K) $^{\text {P range (bar) }}$ \\
\hline MTBE/MeOH & 2 & VLE & 33 & 333.15 & $0.85-1.38$ \\
MTBE/i-butene & 3 & VLE & 16 & $323.15-353.15$ & $0.86-12.24$ \\
MTBE/nC4 & 4 & VLE & 19 & 373.17 & $3.60-15.18$ \\
MeOH/i-butene & 5 & VLE & 11 & 333.14 & $0.55-6.31$ \\
MeOH/nC4 & 5,6 & VLE & 34 & $273.15-373.15$ & $0.04-17.18$ \\
i-butene/nC4 & 7 & VLE & 12 & $277.59-294.26$ & $1.32-2.58$ \\
Acetic acid/isopropanol & 8 & VLE & 19 & $355.55-390.85$ & 1.013 \\
Acetic acid/isopropyl acetate & 9,10 & VLE & 33 & $363.95-388.05$ & 1.013 \\
Acetic acid/water & $11-13$ & VLE & 43 & $373.65-388.49$ & 1.013 \\
Isopropanol/isopropyl acetate & 14,15 & VLE & 61 & $353.65-361.75$ & 1.013 \\
Isopropanol/water & 16,17 & VLE & 32 & $353.34-373.15$ & 1.013 \\
Water/isopropyl acetate & $18-20$ & LLE & 11 & $282.15-347.75$ & 1.013 \\
MTBE/MeOH/i-butene & 21 & VLE & 19 & $333.20-353.20$ & $3.65-11.11$ \\
Acetic acid/isopropanol/water/isopropyl acetate & 22 & VLE & 44 & $356.45-382.65$ & 1.013 \\
\hline
\end{tabular}

${ }^{a}$ VLE: vapor-liquid equilibrium, LLE: liquid-liquid equilibrium

${ }^{b} \mathrm{ND}$ : number of experimental data points

Table S3. UNIQUAC binary interaction parameters employed for MTBE synthesis system $\left(A^{\prime}{ }_{i j}=\right.$ $0)^{23}$

\begin{tabular}{cccccc}
\hline $\boldsymbol{B}^{\prime}{ }_{\boldsymbol{i} \boldsymbol{j}}(\mathrm{K})$ & Methanol & Isobutene & MTBE & n-butane \\
\hline Methanol & 0 & 41.67 & 76.16 & -13.16 \\
Isobutene & -766.91 & 0 & 162.97 & $28.53^{\mathrm{a}}$ \\
$\mathrm{MTBE}$ & -431.66 & -232.67 & 0 & $-148.00^{\mathrm{a}}$ \\
$\mathrm{n}$-butane & -638.06 & $-37.68^{\mathrm{a}}$ & $114.93^{\mathrm{a}}$ & 0 \\
\hline
\end{tabular}

${ }^{\text {a }}$ Determined in this work 
Table S4. UNIQUAC binary interaction parameters employed for acetic acid/isopropanol esterification system ${ }^{23}$

\begin{tabular}{ccccc}
\hline $\boldsymbol{A}^{\prime}{ }_{\boldsymbol{i} \boldsymbol{j}}(-)$ & Acetic acid & Isopropanol & Water & Isopropyl acetate \\
\hline Acetic acid & 0 & 0 & 0 & 0 \\
Isopropanol & 0 & 0 & 2.923 & 0 \\
Water & 0 & -3.313 & 0 & 0 \\
Isopropyl acetate & 0 & 0 & 0 & 0 \\
\hline $\boldsymbol{B}^{\prime}{ }_{\boldsymbol{i} \boldsymbol{j}}(\mathrm{K})$ & Acetic acid & Isopropanol & Water $^{\text {Isopropyl acetate }}$ \\
\hline Acetic acid & 0 & 284.65 & $-539.14^{\text {a }}$ & $188.43^{\text {a }}$ \\
Isopropanol & -301.83 & 0 & $-1133.35^{\text {a }}$ & 67.34 \\
Water & $280.86^{\text {a }}$ & $1106.26^{\text {a }}$ & 0 & -124.82 \\
Isopropyl acetate & $-435.17^{\text {a }}$ & -163.54 & -404.29 & 0 \\
\hline
\end{tabular}

${ }^{\text {a }}$ Determined in this work

Table S5. Extended Antoine parameters ${ }^{23}$ (eq. 27)

\begin{tabular}{cccccc}
\hline Component & $\boldsymbol{a}$ & $\boldsymbol{b}$ & $\boldsymbol{c}$ & $\boldsymbol{d}$ & $\boldsymbol{e}$ \\
\hline Methanol & 71.205 & -6904.5 & -8.8622 & $7.466 \mathrm{E}-06$ & 2 \\
Isobutene & $66.490^{\mathrm{a}}$ & -4634.1 & -8.8975 & $1.341 \mathrm{E}-05$ & 2 \\
MTBE & 45.617 & -5200.7 & -5.1398 & $1.651 \mathrm{E}-17$ & 6 \\
n-butane & 54.830 & -4363.2 & -7.0460 & $9.451 \mathrm{E}-06$ & 2 \\
Acetic acid & 41.757 & -6304.5 & -4.2958 & $8.887 \mathrm{E}-18$ & 6 \\
Isopropanol & 99.207 & -9040.0 & -12.676 & $5.538 \mathrm{E}-06$ & 2 \\
Water & 62.136 & -7258.2 & -7.3037 & $4.165 \mathrm{E}-06$ & 2 \\
Isopropyl acetate & 38.241 & -5563.9 & -3.8789 & $2.476 \mathrm{E}-18$ & 6 \\
\hline
\end{tabular}

a Determined in this work

Table S6. Parameters used in Eq. 30 for the calculation of the equilibrium constant of the MTBE synthesis reaction in the vapor and liquid phase

\begin{tabular}{ccc}
\hline & Vapor phase & Liquid phase \\
\hline $\mathbf{A}_{\mathbf{0}}$ & -11.839 & 1145.292 \\
$\mathbf{A}_{\mathbf{1}}$ & 7649.331 & -14727.568 \\
$\mathbf{A}_{\mathbf{2}}$ & -1.620 & -232.778 \\
$\mathbf{A}_{\mathbf{3}}$ & $1.339 \mathrm{E}-03$ & 1.065 \\
$\mathbf{A}_{\mathbf{4}}$ & $3.343 \mathrm{E}-06$ & $1.077 \mathrm{E}-03$ \\
$\mathbf{A}_{5}$ & $-3.076 \mathrm{E}-09$ & $5.327 \mathrm{E}-07$ \\
\hline
\end{tabular}




\section{References}

1. Hernández-Garduza, O.; García-Sánchez, F.; Ápam-Martínez, D.; Vázquez-Román, R., Vapor pressures of pure compounds using the Peng-Robinson equation of state with three different attractive terms. Fluid Phase Equilib. 2002, 198, (2), 195-228.

2. Toghiani, R. K.; Toghiani, H.; Venkateswarlu, G., Vapor-liquid equilibria for methyl tert-butyl ether + methanol and tert-amyl methyl ether + methanol. Fluid Phase Equilib. 1996, 122, (1), 157-168.

3. Leu, A.-D.; Robinson, D. B., Vapor-Liquid Equilibrium for Four Binary Systems. J. Chem. Eng. Data 1999, 44, (3), 398-400.

4. Wilding, W. V.; Giles, N. F.; Wilson, L. C., Phase Equilibrium Measurements on Nine Binary Mixtures. J. Chem. Eng. Data 1996, 41, (6), 1239-1251.

5. Churkin, V.; Gorshkov, V.; Pavlov, S. Y.; Levicheva, E.; Karpacheva, L., Liquid-vapor equilibrium in C4 hydrocarbon-methanol binary systems. Zh. Fiz. Khim 1978, 52, (2), 488-489.

6. Leu, A. D.; Robinson, D. B., Equilibrium phase properties of the n-butane-hydrogen sulfide and isobutane-hydrogen sulfide binary systems. J. Chem. Eng. Data 1989, 34, (3), 315-319.

7. Martinez-Ortiz, J. A.; Manley, D. B., Vapor pressures for the system isobutane-isobutylene-nbutane. J. Chem. Eng. Data 1978, 23, (2), 165-167.

8. Amezaga, A.; Biarge, F., Equilibrio liquido vapor en sistemas binarios formados por el acido acetico y los alcoholes propilico, isopropilico, isobutilico, butilico secundario y butilico terciario a $760 \mathrm{~mm}$. An. Quim. 1973, 69, 587.

9. Li, X.-l.; Chang, W.-x.; Wan, H.; Guan, G.-f., Vapor-liquid equilibria of isopropyl acetate-acetic acid-water system. J. Chem. Eng. Chin. Univ. 2006, 20, (1), 7.

10. Chang, W.; Guan, G.; Li, X.; Yao, H., Isobaric Vapor-Liquid Equilibria for Water + Acetic Acid + (n-Pentyl Acetate or Isopropyl Acetate). J. Chem. Eng. Data 2005, 50, (4), 1129-1133.

11. Peng, Y.; Liang, J.; Ping, L.; Mao, J.; Zhu, H., Thermodynamic consistency for the vapor-liquid equilibrium data of associating system. Acta Pet. Sin.(Pet. Process.) 2009, 25, 717-724.

12. Zhang, C.; Wan, H.; Xue, L.; Guan, G., Investigation on isobaric vapor liquid equilibrium for acetic acid+water+(n-propyl acetate or iso-butyl acetate). Fluid Phase Equilib. 2011, 305, (1), 6875. 
13. Xie, Q.; Wan, H.; Han, M.; Guan, G., Investigation on isobaric vapor-liquid equilibrium for acetic acid+water+methyl ethyl ketone+isopropyl acetate. Fluid Phase Equilib. 2009, 280, (1), 120-128.

14. Mato, F.; Cepeda, E., Vapor-liquid-equilibrium of binary-mixtures alcohol acetic-acid esters. 1. Systems with methanol, ethanol, normal-propanol and isopropanol. An. Quim. 1984, 80, (3), 338-342.

15. Andreatta, A. E.; Arce, A.; Rodil, E.; Soto, A., Physical properties and phase equilibria of the system isopropyl acetate+isopropanol+1-octyl-3-methyl-imidazolium bis(trifluoromethylsulfonyl)imide. Fluid Phase Equilib. 2010, 287, (2), 84-94.

16. Kojima, K.; Ochi, K.; Nakazawa, Y., Relationship between liquid activity coefficient and composition for ternary systems. Chem. Eng. 1968, 32, 441.

17. Li, Q.; Zhang, J.; Lei, Z.; Zhu, J.; Wang, B.; Huang, X., Isobaric Vapor-Liquid Equilibrium for (Propan-2-ol + Water + 1-Butyl-3-methylimidazolium Tetrafluoroborate). J. Chem. Eng. Data 2009, 54, (9), 2785-2788.

18. Hlavatý, K.; Linek, J., Liquid-liquid equilibria in four ternary acetic acid-organic solvent-water systems at 24.6 ㄷ. Collect. Czech. Chem. Commun. 1973, 38, (2), 374-378.

19. Stephenson, R.; Stuart, J., Mutual binary solubilities: water-alcohols and water-esters. J. Chem. Eng. Data 1986, 31, (1), 56-70.

20. Hong, G.-B.; Lee, M.-J.; Lin, H.-m., Liquid-liquid equilibria of ternary mixtures of water + 2propanol with ethyl acetate, isopropyl acetate, or ethyl caproate. Fluid Phase Equilib. 2002, 202, (2), 239-252.

21. Vetere, A.; Miracca, I.; Cianci, F., Correlation and prediction of the vapor-liquid equilibria of the binary and ternary systems involved in MTBE synthesis. Fluid Phase Equilib. 1993, 90, (1), 189-203.

22. Lee, L.-S.; Kuo, M.-z., Phase and reaction equilibria of the acetic acid-isopropanol-isopropyl acetate-water system at 760 mmHg. Fluid Phase Equilib. 1996, 123, (1), 147-165.

23. Aspen Plus v8.8, Aspen Technology Inc.: Cambridge, MA, 2014. 